\title{
The Design of an Embedded Framework for Programmable Automation Systems
}

\author{
Ramin Ardekani ${ }^{1}$, Kevin Oldknow ${ }^{2}$ and Ian Yellowley ${ }^{2}$ \\ ${ }^{1}$ Cameleon Controls Inc., ${ }^{2}$ Department of Mechanical Engineering, University of British Columbia \\ yellowley@mech.ubc.ca
}

\begin{abstract}
The authors describe the development of a simple embedded framework that provides the basis for the design of customised programmable automation systems for a variety of applications. The framework provides the user with a standard environment for system development that encompasses motion, plc, hmi, communication and general monitoring/control applications. The system can be extended to provide plug and play facilities that in turn facilitate automated reconfiguration for system extension or modification. Examples of the use of the system and potential extensions to new areas are provided
\end{abstract}

\section{Introduction}

The programming of complex automation systems usually involves the integration of a series of different hardware and software systems, each developed to a highly specialized state to suit their particular application. Typical automation applications will require motion control, hmi, and plc activities to be integrated, and in most cases the processes themselves can benefit from more basic work on monitoring, control or signal conditioning. The monitoring and control applications themselves will often be written in different languages and may need special hardware. While it is clear that the languages and hardware used have been optimized about their specific applications, their structure may detract from the overall efficiency of the end product and certainly the process of integration is often made extremely difficult, especially if an oem wishes to select software and hardware from a variety of vendors. Even when a single vendor is used, later updating and extension of the system may prove difficult.

The design project described here has been ongoing for many years. All the initial work was carried out within the Manufacturing Engineering Laboratory at UBC and involved the design of open architecture CNC systems, (high performance motion, PLC and monitoring functions). Later commercial versions of the framework have been developed by Cameleon Controls for general motion, plc and hmi applications. The project was undertaken initially to design and develop specific tools and more particularly broad frameworks that would provide a base upon which complex automation projects may be quickly and easily built. The tools and framework designed and built to this stage achieve many of the benefits originally aimed for. The methodologies applied are unusual or perhaps more correctly "non mainstream" The magnitude of the benefits that may be achieved in the end is dependant on the willingness of a range of automation companies to standardize their interfaces and software structures in a manner that presents the user with a uniform open programmable interface to their products.

\section{The Basic Problem}

The main design requirements of programmable automation systems can be roughly classified as follows:

a) Performance and cost

b) Flexibility and ease of use

c) Vendor neutrality at specific levels of granularity.

d) Degree of openness to reprogramming and insertion of new (improved) hardware and software tools.

It seems that the rather academic notions of openness have (thankfully) disappeared, however the day be day reality of being able to modify, extend systems and integrate additional components without detracting from system performance is still a central issue in the design of the modern automation systems.

As mentioned in the introduction, several of the constituent areas have highly specialized hardware and specific software tools, and these have been developed to program these specialised sub-systems over many years. The result of this is a tendency to force system designers to think of openness at a very large level of granularity, and usually at high levels of the applications. This approach is generally inefficient, especially if one is designing systems where there is a significant need for coupling between the various subsystems. There are many possibilities in terms of the design of an integration strategy and clearly each has advantages to different clients, two extremes may be envisaged at the early stage of conceptual design to guide progress. 
a) The building of middleware comprising programs and databases to permit these specialized subsystems to cooperate with each other. This is the most popular approach and indeed there are several attempts to provide such interfaces already on the market. It is not usual for vendors to provide access to lower level software through this mechanism but certainly it could be done.

b) The building of a complete foundation framework that allows access to all functions, including the lower level operating system or communication functions. This by definition would be programmed to provide access to lower level system services in all applications

The crux of the issue really is the extent to which efficiency and use considerations mandate system integration; this depends in large part upon the structure of the environment and the ambitions of the oem. The oem is the focus of this work and the authors are aware that the oem interest may very well be opposed to those of the individual software and hardware component manufacturers. The issues revolve around the degree of granularity in the software, (and hardware), and the accessibility of the process to integration at each level of granularity. It is clearly easier to control performance if one is able to encapsulate all performance-influencing factors in such a way as to prevent other applications from impacting performance, even though this may not be of benefit to individual sub-system performance. In a normal system where individual applications are called by some type of run time environment and share some central shared interface it is difficult to sequence and schedule activities without low level application specific knowledge of the application, the operating system and the individual software tools that are part of the system.

It has long been realized that abstraction is a useful tool in the building of efficient programming systems. The programmable automation environment benefits from abstraction as far as the physical computing environment is concerned but in this case the programmer is often a process expert and benefits greatly or perhaps even requires closeness to the actual automation hardware. One thus has an interesting situation where one wishes to be able to access specialized automation hardware and the process being controlled in an intimate fashion, but still abstract the middle layer used to achieve the majority of the computation. One expects that by building an abstracted middle layer that provides the majority of the computational functionality then the amount of new programming and the uniformity of the approach can both be controlled while providing an easy to use environment for the programmer.

To achieve all required aims, maintain generality, and still provide an efficient environment for the design of specialized applications, one is drawn towards a system with 3 levels of operation:
1) A specialized virtual machine environment where the programmer is in close control of the physical automation components.

2) A generalized framework that is able to provide simple abstracted access to general purpose platform-dependant computing facilities, (computing, communication, $\mathrm{i} / \mathrm{o}$, etc). This layer should be as comprehensive as is possible without overlap with automation or process specific functions. It will comprise approximately $80 \%$ of the code in the typical final application.

3) A set of low level drivers implemented by the component supplier that provides the specific hardware dependant methods needed to achieve the specific operations requested by either the virtual machine environment or the general framework. In the scheme suggested by the authors this code is expected to be resident on module or physical component.

Step 3 is particularly critical to the commercial success of the approach, and follows naturally from the technical approach taken. It allows easy object based abstraction at step 2 and at the same time provides complete vendor neutrality, flexibility and automated configuration to be applied if required. The approach taken here builds on similar approaches to the configuration of generalised computing units, noteworthy here is the Open-Firmware initiative initially developed by SUN Microsystems and standardized as IEEE1275 (1), as Open_Boot and several closely related initiatives, (2), (3).

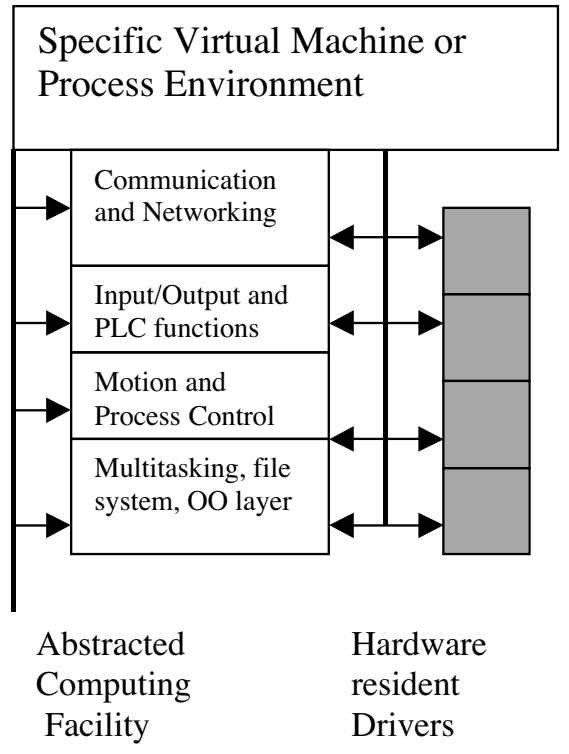

Figure (1) Virtual machine abstracted framework and hardware resident drivers 
In essence one builds an abstracted computing facility by carrying specific hardware dependant methods on module, (akin to drivers in the PC world), and by

having highly abstracted and likely highly object oriented environments at the higher level to describe an equivalent virtual environment. All that is then needed is a mechanism to ensure that the correct real methods are attached to the virtual methods at run time.

The method chosen here was first developed for machine tool CNC systems, (4) and is called Open_Config.

The basic Open_Config environment was initially applied to $\mathrm{CNC}$ systems, the high level environment then is a rather specialized motion control environment with machine tool specific features to suit the CNC application. The hardware modules in this case are FPGA based controllers that implement the highly parallel UBC control architecture shown in Figure (2)

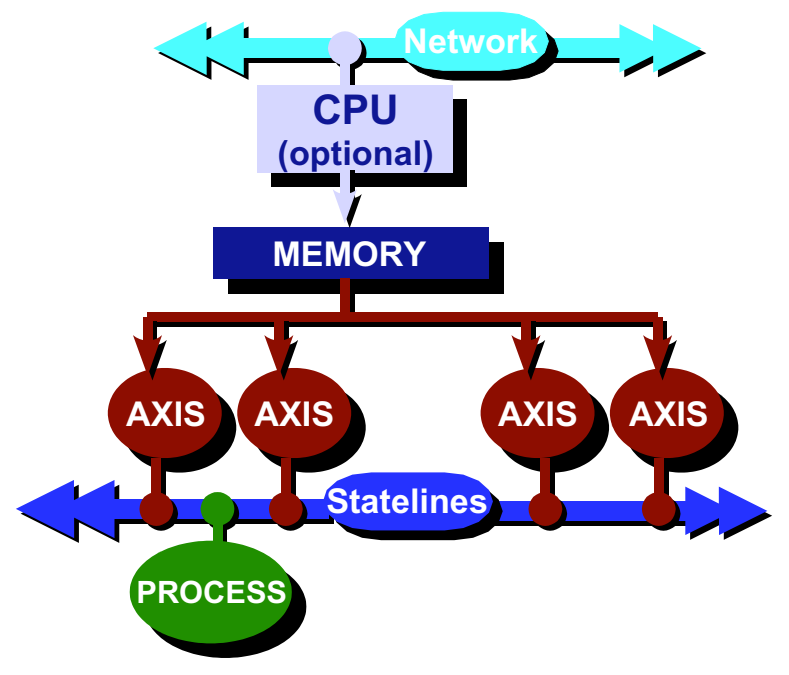

Figure (2) Highly parallel control architecture

The issue of course is how one integrates the required extensibility into the system. The solution in this case is to use the FORTH programming language at the abstracted framework level in similar fashion to Open Firmware. This then provides a simple extensible, interactive language facility and the capacity to add methods and change the code corresponding to methods in a manner that would be cumbersome in other languages. The actual mechanisms used are a little different to those in other firmware systems. The firmware layer and the virtual machine layer both have a series of objects and methods that describe the complete automation and computational environments. The virtual methods allow the driving of virtual environments only. The connection between the virtual and physical e code on the system components to virtual methods through a simple binding table that passes the execution token of the required method to the virtual method. The overall system is shown in Figure (3) below, the nature of the binding allows many advantages in terms of system reconfiguration in real time, and the use of this facility in the building of safety critical systems.

The basic ideas of Open_Config are fairly easy to appreciate. The building of complete frameworks that permit automation companies to design real world systems in a simple robust manner using suc a facility is non trivial. The remainder of this paper discusses the design of such a system based on Open_Config, the work was carried out by Cameleon Controls with support from UBC. The resulting environment is termed the embedded Cameleon Application framework, (eCAF) and has been applied to various modular industrial electronic systems. In each case the virtual part of the abstracted computing environment remains mainly constant. To demonstrate the use of the framework the authors will discuss the motion system gui developed by Cameleon Controls that provides a clear view of a typical virtual machine environment designed to sit on top of $e C A F$.

\section{The Embedded Cameleon Application Framework, $(e C A F)$}

The $e C A F$ system is shipped by Cameleon Controls as a complete embedded development framework. Where there is a cooperation with a hardware vendor then the system dependant methods are stored on module, where this is not the case application level tools are held on a master processor and instantiated once a particular module is recognized as present in the application.

In order to cover the range of applications needed, the framework has a broad variety of classes each with a rich vocabulary of methods. The idea of course is not just to abstract the physical hardware but to reduce the amount of coding required of the programmer. It is not possible here to give a complete description of the framework but the authors will give an overview and some details of the simplest classes

Typically the framework provides a range of communication options over TCP/IP, CAN and simple serial protocols are almost always present, as are some higher level protocols such as Modbus and many systems add specific local I/O options to support particular vendors. The I/O usually comprises a set of methods to sample or drive digital and analog I/O channels as well as specific routines to count or to decode quadrature signals or to filter specific signals etc. $e C A F$ is a very much motion centric system so the control of high performance multi-axis motion is supported, typical methods of the axis and interpolator classes are listed in an Appendix and should be self explanatory. 


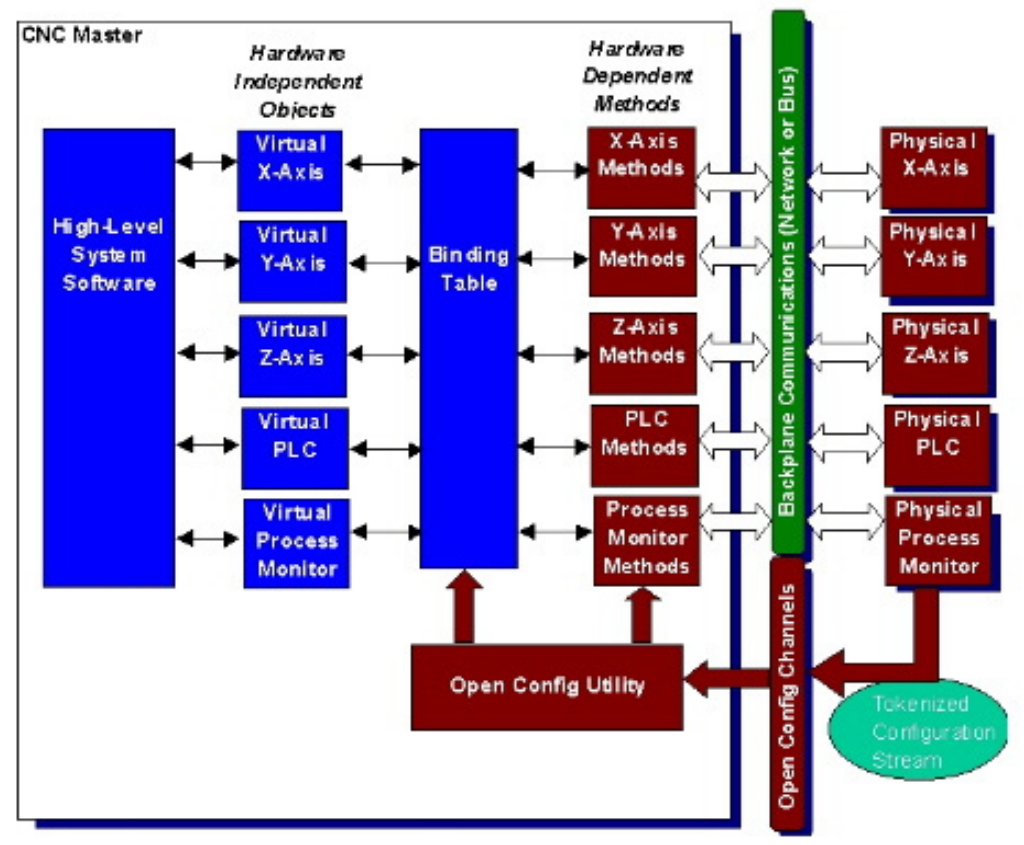

Figure (3) Open_Config a configuration system for programmable automation

The system builds on operating system facilities to perform activities such as file management and multitasking, to make this more straightforward then the FORTH version selected for $e C A F$ is written in ANSI C, this means that new FORTH words are built in either C or in FORTH and both portability to new CPU's and addition of operating system facilities to the core are both easily achieved. The specific FORTH used is the Portable Forth Environment, (PFE), it may be noted that alternatives such as the Forth Inspired Command Language (FICL) developed as the firmware layer for FreeBSD or GForth would make suitable alternatives, each with slightly different characteristics. One can see then that the framework tries to give easy access to all facilities without having to switch between applications, often used functions, (like file transfer for instance), are embedded in the framework and create a much more productive environment. A critical part of the strategy is to ensure programmability. The framework uses a resident FORTH interpreter, typically running on a low priority thread within a multitasking operating system to achieve this end. The interpreter provides the possibility for the programmer to action methods locally or remotely, but more importantly it also allows the system to compile new code to be run at a later time using the interpreter and text strings passed to it from a variety of sources.

The complete overall structure of the approach taken can now be shown in Figure (4). The three layer approach allows almost unlimited customization. As mentioned earlier customiation can be based on the use of standard classes to represent specific application specific objects, at the same time though the system is amenable to the definition of new application specific classes at the highest level. These can still use the whole system including Open_Config and the command interpreter but present completely different capabilities through their own methods that reach through the standard framework. The customization possibilities are illustrated in Figure (5). Before describing the high level use of the system a very simple class will be briefly described.

\subsection{An example of a Simple Class (Digital I/O)}

The following base digital i/o class files are held within the framework:
a. digital_input.cls
b. digital_output.cls
c. pwm_output.cls

$e C A F$ instantiates a set of standard input/output objects at boot time using these generic classes:

a. digital inputs, $\operatorname{din} 0 \ldots \operatorname{din} 7$

b. digital outputs, dout 0 ... dout7

c. pwm outputs, pwm0 .. pwm0 


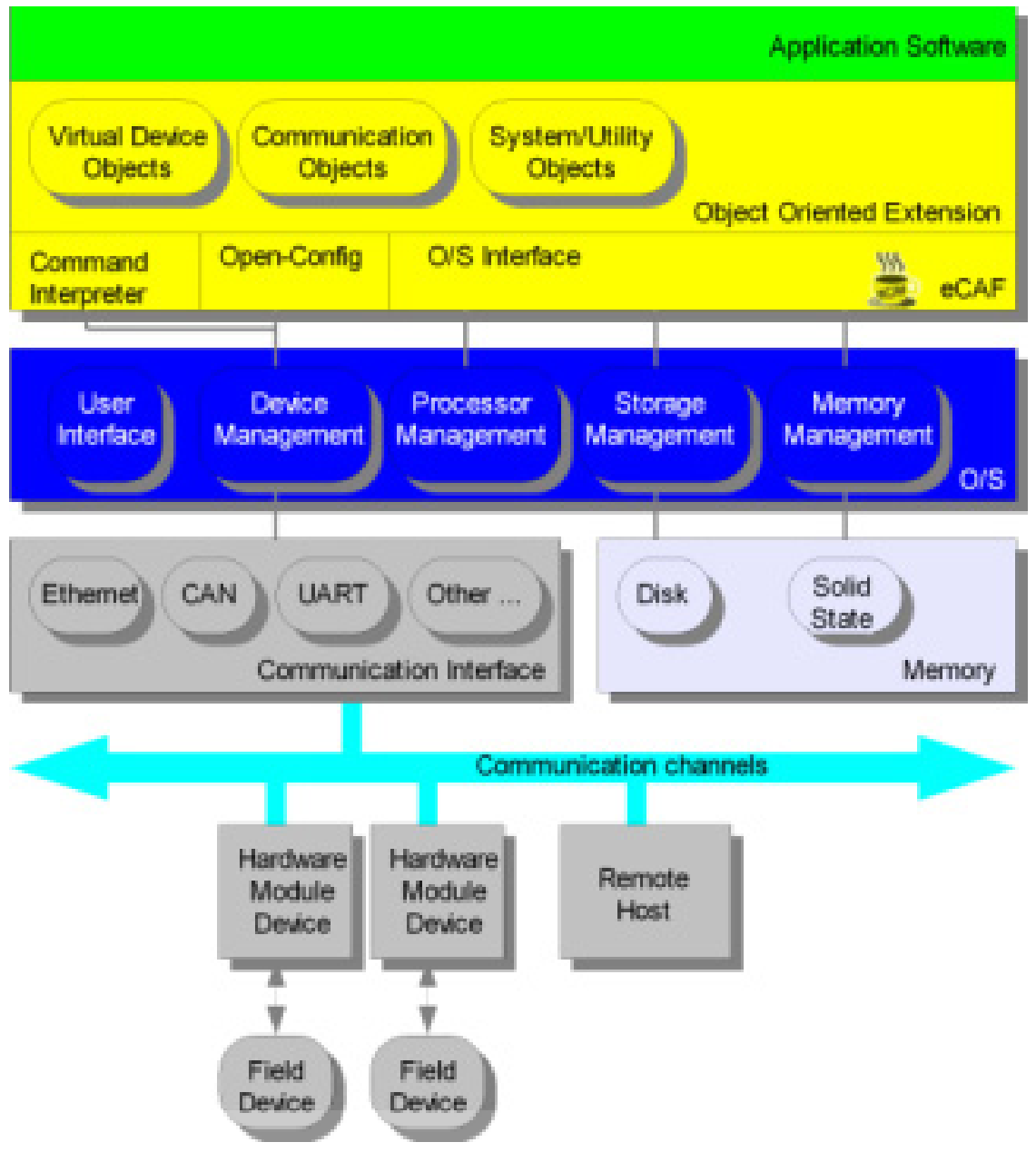

Figure (4) Overall 3 layer software scheme

The virtual methods for these objects are registered in the I/O binding table and are bound to device methods at boot time.

Classes that are more application specific, such as (say) push_button, limit_switch, selector_switch, ouput relay etc. can also be built. Application specific vocabulary within the higher virtual machine environment can then be generated by instantiating objects using these classes. For example a push_button class could be used to instantiate estop and actuate objects on a CNC pendant; a limit switch class used to instantiate xlimit + and xlimit- axis travel limits. I/O modules programmed with a device file corresponding to these object virtual methods would simply bind hardware device methods to associated application object methods at boot time.

Methods supplied by $e C A F$ to interact with these objects include the following (self explanatory) examples. Methods typically leave an I/O error flag on the interpreter stack. A successful I/O operation is indicated by a 0 flag. set:

show:

reverse polarity:

clear:

Other less obvious methods are available to locate hardware addresses to toggle outputs etc. and the system wide methods like show: or version: can be used to examine the methods available or version being used respecitively and output polarity is reversed with the reverse polarity: method. PWM methods again are fairly self explanatory, for instance, the code below generates a $16 \mathrm{~Hz}, 50 \%$ duty cycle pwm signal,

$16 \mathrm{hz}$ freq: $\mathrm{pwm} 0$

50\% duty: pwm0 enable: pwm0 


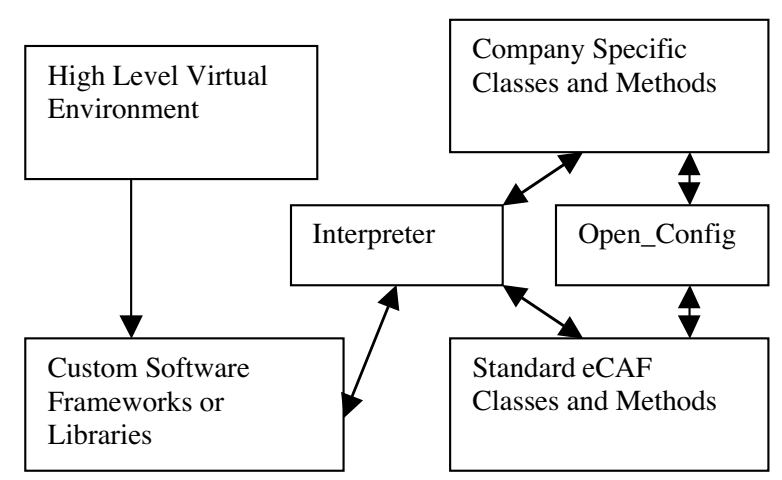

Figure (5) System customisation

\section{Example of High Level Application Development}

The example used to illustrate a typical high level application is the development of an intelligent graphical system for servo tuning and test; the system was developed by Cameleon controls. The graphical environment connects to $e C A F$ over TCP/IP (usually $e C A F$ is running on a separate embedded processor and the gui on a host PC, but both could be resident on a single processor). The high level environment is written in Python using wxWindows to leverage the libraries and ease of constructing complex windowed environments. (Cameleon has also produced simple environments using Java, they seem to require significantly more effort).

The Python environment provides a tremendous amount of help in terms of building the gui, it allows considerable flexibility in the customization of the system with relatively little effort. The environment directly leverages all the power of standard $e C A F$ classes and some Cameleon specific classes and methods by passing strings directly through the interpreter. This simple technique allows the use of all motion, data collection and communication classes leaving the Python environment responsible for input and display functions.

The basic gui is shown in Figure (6). The total environment encompasses the setting of communication parameters, the setting of motion parameters and more specialized environments to allow the tuning of various types of servo and finally to perform 2 axis interpolation in a simplified $\mathrm{CNC}$ interface.

The initial environment is designed to allow easy setup of communications and includes an automated system software update procedure. Typically eCAF will also allow a parallel debug facility running over a serial port or over tcp/ip. In this case the port will report the success or failure of the system to complete the actions requested as shown in Figure (7).

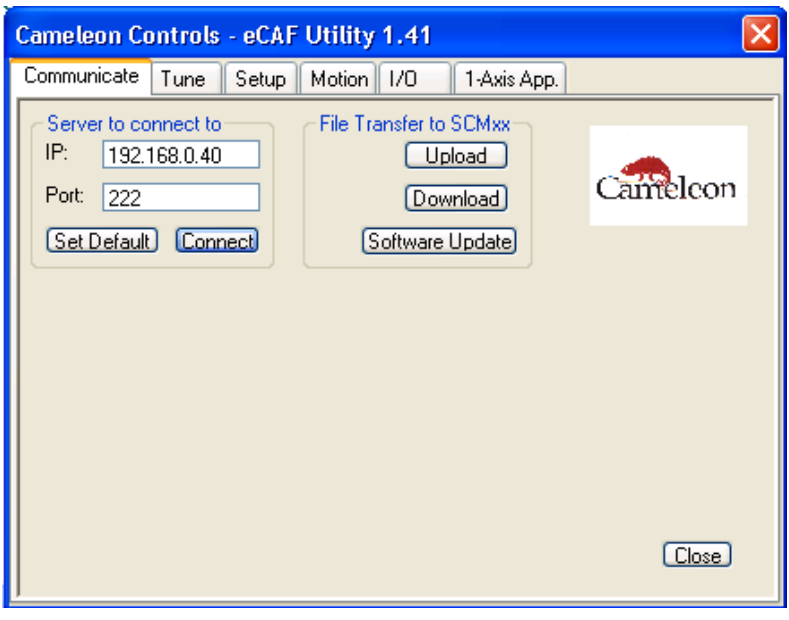

Figure (6) High level servo tune and test facility

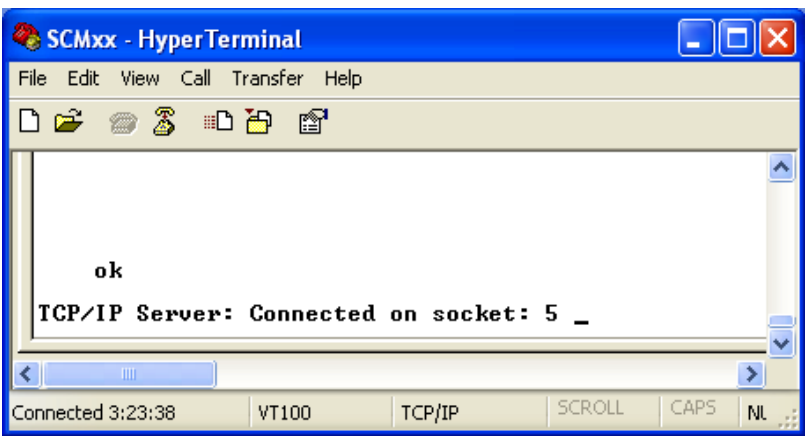

Figure (7) Hyperterminal connection to eCAF

The more complex tasks of axis tuning use the $e C A F$ classes to control input to the amplifier, to measure motor response and display this for the user, again readers can get an appreciation for the range of motion classes from the Appendix. The usual gui for torque mode tuning is a two stage process the fine control stage is depicted in Figure (8) A sinusoidal input is used in the coarse stage followed by a step input in the second fine stage. The data recorder classes from $e C A F$ are used to collect data and the result plotted as shown.

Finally the motion gui itself allows a user to drive two axes in linear and circular modes while displaying position and other parameters. This interface is relatively easily extended to become a full $\mathrm{CNC}$ interface using facilities provided by $e C A F$ in the macro class.

The point of the example is to demonstrate that a very customised and complex application can be built very quickly and can drive proprietary functionality while leveraging the vast majority of the code from a standard framework. 


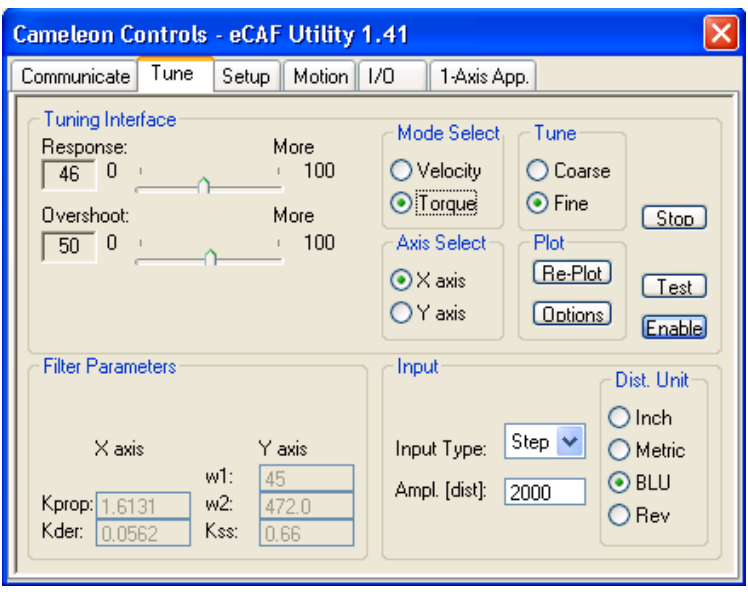

Figure (7) Fine tuning mode

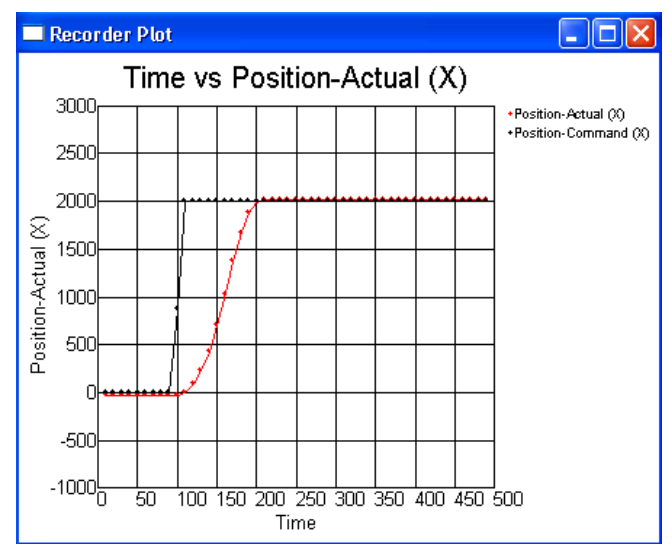

Figure (8) Motor response

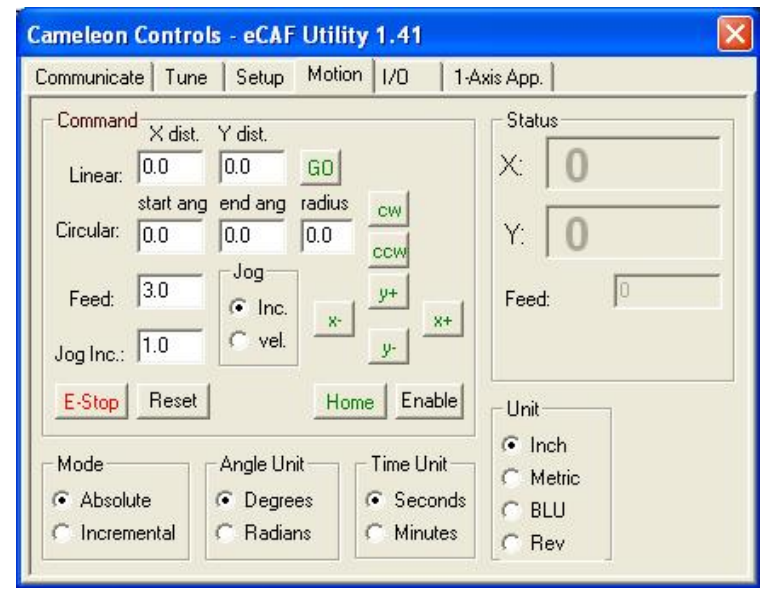

Figure (9) General 2 axis motion interface
Configuration) Firmware: Core Requirements and Practices - IEEE Std. 1275-1994. Institute of Electrical and Electronics Engineers, New York. 2. Caffrey, R. Shaw, H. and Wagner, L. (1997) Developing plug-and-play spacecraft systems: NASA Goddard Space Flight Center's (GSFC) Essential Services Node (ESN). AIAA-IEEE Digital Avionics Systems Conference Proceedings 1:2.1.28-2.1.35.

3. Eckert, B. (1999) Firware Factory User's Manual. [resource on-line]; available from http://www.geocities.com/SiliconValley/Cable/3439/ 4. Oldknow, K.D. and Yellowley, I. (2001) Design, implementation and validation of a system for the dynamic reconfiguration of open architecture machine tool controls, International Journal of Machine Tools \& Manufacture, 41:795-808

\section{Appendix 1. Motion Classes and Methods}

INTERPOLATOR Methods, (Coordinated Motion, Linear and Circular modes)

describe: version: resume: suspend: show: set_delay: set priority: get_priority: get_delay: get_status: get_thread: lin: cw: ccw: set_feed: get_master_prd: set_accel: get accel: set incremental set_absolute: get_move_mode: set_distance_mm: set_distance_inch: set_distance_rev: get_distance_unit: set_time_sec:

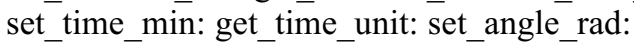
set_angle_deg: get_angle unit: home: put_radius: get_radius: put_direction: get_direction: put_sangle: get_sangle: put_eangle: get_eangle: dwell: abort: get_busy?: get_moving?: feed_limit_on: feed_limit_off: get_feed_limit:

\section{AXIS Methods (basic single axis, control and kinematic settings)}

describe: version: show: get_btable: Register: set_mm/rev: get_mm/rev: set_BLU/rev: get_BLU/rev: get_BLU/dist: get_min_distance: set_min_distance: get_max_distance: set_max_distance: get_max_velocity: set_max_velocity: get_velocity: set_velocity: get_ref_position: set_ref_position: set_in_position_range: get_in_position_range: set_radius_dist: get_radius_BLU: put: get: clear: check_status: get_ts: get_tm: set_filter: reset: halt: enable: send_increment: read_position: read_error: flush_buffer: get_buffer_stat: get_buffer_count: zero_on_next_z: zero_in_progress? in_position?: get_pwm_output: set_pwm_output: get_msratio: send_header: set_lcmode: get_lcmode: get_lcfreq: set_lcfreq: get_seq_num:

\section{Bibliography}

1. Bradley, W.M., Kahn, D.M., Rible, J. and Williams, M. (1994) IEEE Standard for Boot (Initialization 